\title{
Evaluación de Tecnologías Sanitarias: Fascitis plantar, epicondilitis y hombro doloroso: tratamiento con ondas de choque extracorpóreas
}

\section{Contexto clínico}

Las ondas de choque extracorpóreas (OCHE) fueron utilizadas inicialmente en medicina como una forma de fragmentación de los cálculos renales para facilitar su excreción urinaria'. En relación a las afecciones musculoesqueléticas se supone que existe un depósito de microcalcificaciones en las inserciones ligamentosas o musculares, lo que se relacionaría al dolor, con la consecuente disminución del mismo al facilitar su disolución. Entre las afecciones musculoesqueléticas que se beneficiarían con OCHE se encuentra la fascitis plantar, la epicondilitis y el hombro doloroso.

Fascitis plantar: dolor principalmente en el talón que se irradia hacia la planta del pie. Sucede con mayor frecuencia al levantarse y cargar peso y afecta al $10 \%$ de la población en algún momento de su vida ${ }^{2}$. Resultaría de una inflamación de las inserciones musculares y ligamentosas (entesitis) en el calcáneo y de las almohadillas vasculares de la planta del pié. Es habitualmente autolimitada pero en algunas personas el dolor tiene una intensidad tal como para interferir en sus actividades habituales.

Epicondilitis: dolor en el epicóndilo de la articulación del codo que puede estar asociado a dolor en la dorsiflexión bajo resistencia de la articulación de la muñeca. Su prevalencia es del 1 a $3 \%$ y su pico de incidencia, entre los 40 y 50 años. Su incidencia en atención primaria es de 4 a 7 casos cada 1000 personas por año ${ }^{3}$ y resultaría de una lesión menor pero sostenida del grupo muscular extensor del antebrazo. Es generalmente autolimitada pero algunas personas pueden persistir con dolor por más de un año.

Hombro doloroso: dolor en las articulaciones glenohumeral, acromioclavicular, esternoclavicular, subacromial, escapulotorácicas y los tejidos blandos circundantes. Independientemente de la etiología, generalmente el motivo de consulta es el dolor. Su prevalencia oscila entre 5 y $20 \%$ y el pico de incidencia se da entre los 45 y 70 años. Resulta de algún trastorno del manguito rotador en el $70 \%$ de los casos. La mitad de los casos evoluciona favorablemente al año de comenzado, persistiendo algunas personas con dolor durante tres años ${ }^{4}$.

Actualmente existen tres dispositivos aprobados por la Administración de Drogas y Medicamentos de EEUU (en inglés FDA) para su uso en pacientes con estas afecciones que persistan con dolor por mas de seis meses y no hayan respondido a los métodos terapéuticos convencionales ${ }^{5}$.

\section{Objetivo}

Evaluar la efectividad de las OCHE en la fascitis plantar, la epicondilitis y el hombro doloroso.

\section{Descripción de la tecnología}

Las ondas de choque extracorpóreas (OCHE) consisten en la generación de ondas acústicas de alta frecuencia fuera del cuerpo, que mediante un sistema de amortiguación -antiguamente agua y actualmente almohadillas- se pueden dirigir y focalizar en áreas específicas. Se administran en sesiones de 15 a 20 minutos sin analgesia o en algunos casos, analgesia leve. Actuarían en estructuras con algún componente cálcico a través de gradientes de presión transitorios, lo que facilitaría su disgregación y posterior absorción por el organismo.

\section{Métodos}

Búsqueda en bases de datos específicas (MEDLINE, DARE, LILACS) y en un buscador genérico. Los términos de búsqueda fueron "musculoskeletal Diseases" y "extracorporeal shock wave Therapy (ESWT)".

\section{Resultados}

Se hallaron 44 publicaciones, de las cuales se seleccionaron 25 (cinco revisiones sistemáticas, 15 ensayos clínicos controlados aleatorizados que no habían sido incluidos en las revisiones sistemáticas, dos evaluaciones de tecnologías y tres informes de políticas de cobertura).

Fascitis plantar: dos revisiones sistemáticas ${ }^{2,6}$ y cuatro artículos no incluidos en estas revisiones. Salvo en uno de los artículos seleccionados que informa una diferencia significativa evaluada mediante una escala analógica visual al mes y a los tres meses, ambas revisiones sistemáticas y la mayoría de los ECCA seleccionados no encontraron diferencias significativas entre OCHE y placebo.

Epicondilitis: dos revisiones sistemáticas ${ }^{8,3}$ y cinco artículos no incluídos en estas revisiones que no mostraron que la OCHE sea mejor que el placebo. Un ensayo clínico comparó la OCHE con la aplicación de corticoides intrarticulares y encontró una diferencia a favor de estos últimos (84-60\% a los tres meses).

Hombro doloroso: una revisión sistemática ${ }^{4}$ y seis ECCA no incluidos en esta. La OCHE de alta energía contribuiría a la mejoría de los síntomas relacionados al hombro doloroso (grado de dolor, movilidad y limitación de actividades) especialmente en pacientes con tendinitis calcificada.

\section{Conclusión}

Ninguna revisión arrojó resultados concluyentes acerca del beneficio de las OCHE. De los 15 ECCA, solamente uno en hombro doloroso mostró resultados beneficiosos. Dos encontraron resultados estadísticamente significativos pero con escasa significancia clínica y el resto no mostró beneficios. No se halló evidencia de que la OCHE confiera claros beneficios en alguna de las indicaciones analizadas. La mayoría de las organizaciones de salud analizadas considera a las OCHE un procedimiento en fase de investigación. Debido a algunos resultados contradictorios, especialmente en pacientes con hombro doloroso (tendinitis calcificada del supraespinoso) esta indicación deberá ser evaluada en futuros ensayos clínicos.

Modificado de: García Martí S. Utilidad de las ondas de choque extracorpóreas en las afecciones musculoesqueléticas. Instituto de Efectividad Clínica y Sanitaria (www.iecs.org.ar). Documentos de Evaluación de Tecnologías Sanitarias, Informe Técnico Breve no. 15. Buenos Aires, Argentina. Agosto de 2004.

\section{Referencias}

1. Chung B, Preston WJ. Extracorporeal Shockwave Therapy. A Review. Sports Med 2002; 32 (13): 851-865.

2. Crawford F, Thomson C. Interventions for treating plantar heel pain. Cochrane Database Syst Rev.2003;(3).

3. Assendelft W, Green S, Buchbinder R. Tennis elbow. Clin Evid 2003; 10:1493-1503.

4. Speed C, Hazleman B. Shoulder Pain. Clin Evid 2003; 10:1477-1492.

5. Extracorporeal Shockwave Therapy for Musculoskeletal Indications. Technology Assessment Update Office of the Medical Director Department of Labor and Industries. March $29,2004$.

6. Crawford F. Plantar heel pain and fascitis. Clin Evid 2003; 10:1431-1443.

7. Cosentino R, Falsetti P. Efficacy of extracorporeal shock wave treatment in calcaneal enthesophytosis. Ann Rheum Dis. 2001 Nov; 60(11): 1064-7.

8. Buchbinder R, Green S, White M. Shock wave therapy for lateral elbow pain. Cochrane Database Syst Rev. 2004:(1).

9. Crowther MA, Bannister GC. A prospective, randomised study to compare extracorporeal shock-wave therapy and injection of steroid for the treatment of tennis elbow. J Bone Joint Surg Br. 2002 Jul; 84(5):678-9. 